Check for updates

Cite this: RSC Adv., 2019, 9, 26091

Received 10th July 2019

Accepted 15th August 2019

DOI: $10.1039 / c 9 r a 05264 j$

rsc.li/rsc-advances

\section{Monomer sequence design at two solvent interface enables the synthesis of highly photoactive carbon nitride $\uparrow$}

\author{
Susmita Dolai, $₫$ Neeta Karjule, $\$$ Adi Azoulay and Jesús Barrio (iD *
}

Structural modifications in carbon nitrides and related carbon-based materials have been achieved in recent years by organizing their monomers into versatile supramolecular structures that serve as reactants for the high temperature solid-state reaction. To date, the organization is usually carried out in one solvent where the building blocks must be dispersed. Here, we show the utilization of a molecule with both hydrogen bond donor and acceptor sites for constructing hydrogen bonded frameworks in interfacial systems. The chemical and electronic properties of the carbon nitride materials after calcination are strongly altered showing enhanced photocatalytic performance in different model reactions. This work shows a new large-scale pathway for the synthesis of highly photoactive carbon nitride with tailored properties and morphology by employing novel supramolecular assemblies prepared in the interface between two solvents, and furthermore opens new opportunities in the rational design of different carbon-nitrogen based materials utilizing supramolecular structures.

\section{Introduction}

The tunable chemical and electronic properties as well as high stability and low cost of polymeric carbon nitride $(\mathrm{CN})^{1,2}$ allow its exploitation in many fields including photo- and electrocatalysis, ${ }^{3-5} \quad \mathrm{CO}_{2}$ reduction, ${ }^{6,7}$ water treatment, ${ }^{8,9}$ sensing,$^{10,11}$ LEDs, ${ }^{12}$ and more.$^{13,14}$ Despite this wide research, tailoring its chemical and photophysical properties has represented a continual challenge due to its solid-state synthesis, which yields unordered materials with low photoactivity. To overcome these drawbacks, doping with heteroatoms, ${ }^{15,16}$ the use of hard and soft templates, ${ }^{17,18}$ and the formation of heterojunctions with different materials ${ }^{19-22}$ have been utilized in order to improve $\mathrm{CN}$ surface area, ${ }^{23}$ as well as to promote charge transfer and separation properties and overall catalytic activity. ${ }^{24}$ In the past six years, the use of ordered supramolecular structures comprised of C/N monomers has emerged as an alternative approach for synthesizing CN, nitrogen-doped carbon and carbon materials for various applications..$^{25} \mathrm{~A}$ tailored monomer sequence permits the design of electronic structures with enhanced light harvesting properties ${ }^{26,27}$ or novel and ordered morphologies without the need of templating methods like hollow

Department of Chemistry, Ilse Katz Institute for Nanoscale Science and Technology, Ben-Gurion University of the Negev, Beer-Sheva 8410501, Israel. E-mail: barriohe@ post.bgu.ac.il

$\dagger$ Electronic supplementary information (ESI) available. See DOI: 10.1039/c9ra05264j

\$ Susmita Dolai and Neeta Karjule contributed equally to this work. tubes, ${ }^{28,29}$ rods, ${ }^{30,31}$ spheres, ${ }^{32,33}$ or dots $^{34,35}$ with enhanced number of active sites and overall enhanced photocatalytic activity. ${ }^{36}$ Our group and others have identified the importance of solvents in the supramolecular assembly as well as in the final $\mathrm{CN}$ formation and properties such as morphology, size and activity. ${ }^{37,38}$ Recently we demonstrated that a sequential treatment of supramolecular structures with different solvents can produce either a complete rearrangement or just surface modifications depending on the polarity of the solvent. ${ }^{39}$ Furthermore, we illustrated that the interfacial supramolecular organization on two non-miscible solvents $^{40}$ leads to a significant modification of all $\mathrm{CN}$ parameters, e.g., morphology, surface area, and photophysical properties. ${ }^{41}$ In this work we show the design of unique supramolecular assemblies in a water/chloroform system using melamine, ${ }^{42}$ cyanuric acid, ${ }^{43}$ and the amino acid creatine. ${ }^{44}$ The starting monomers will be preorganized in a water-chloroform interfacial system, where creatine selectively binds melamine units in the water phase acting as hydrogen bond acceptor, and also the cyanuric acid moieties in the chloroform phase, acting as hydrogen bond donor. ${ }^{1} \mathrm{H}^{-}$ NMR, XRD and FT-IR have proven the formation of hydrogenbonded frameworks and the selective binding of creatine to both melamine and cyanuric acid units in the interface. At high temperatures creatine condensates to guanidine-like structural motifs that interlink the heptazine units and alter the photo-physical and morphological properties of the materials leading to an enhanced conductivity and photocatalytic activity in the hydrogen evolution reaction and the degradation of rhodamine B dye. 


\section{Experimental section}

\section{Synthesis of CN materials}

$630 \mathrm{mg}(5 \mathrm{mmol})$ of melamine were dispersed in $25 \mathrm{~mL}$ of deionized water and different amounts of creatine were added (32, 64 and $168 \mathrm{mg}, 0.25,0.5$ and $1.25 \mathrm{mmol}$ respectively) forming melamine-creatine complexes (M-C). Afterwards, a dispersion of cyanuric acid in chloroform (645 $\mathrm{mg}, 5 \mathrm{mmol}$ in $25 \mathrm{~mL}$ ) was added to the $\mathrm{M}-\mathrm{C}$ suspensions, and the formation of modified cyanuric acid-melamine complexes $(\mathrm{CM})^{45}$ happens at the interface between the two solvents. The solids were filtered, washed with distilled water, dried at $60{ }^{\circ} \mathrm{C}$ in vacuum overnight and labelled as $\mathrm{CMC}_{x}$ (were $x$ corresponds to the amount of creatine in molar ratio versus the cyanuric acid and melamine, namely $0.05,0.1$ and 0.25 ). The resulting powders were calcined at $550{ }^{\circ} \mathrm{C}$ for $4 \mathrm{~h}$ (heating rate of $2.3{ }^{\circ} \mathrm{C} \mathrm{min}^{-1}$ ) under an inert nitrogen atmosphere in order to obtain modified carbon nitride materials $\left(\mathrm{CMC}_{x}-\mathrm{CN}\right)$. A reference material was synthesized by following the same procedure in the absence of creatine (CM-CN).

\section{Characterization}

Scanning electron microscopy (SEM) images, energy dispersive X-ray (EDX) spectroscopy and elemental mapping were obtained using a JEOL JSM-7400F system operated at $3 \mathrm{kV}$ equipped with Thermo Scientific NORAN System SIX. Transmission electron microscopy (TEM) images were recorded using a FEI Tecnai T12 G2 TWIN transmission electron microscope operated at $120 \mathrm{kV}$. A JEOL JEM-2100F analytical TEM operated at $200 \mathrm{kV}$ as used for high-resolution transmission electron microscopy (HRTEM) imaging. X-ray diffraction patterns (XRD) of the synthesized powders were obtained using an Empyrean powder diffractometer (Panalytical). The Fourier-transform infrared spectroscopy (FTIR) analysis was performed on a Thermo Scientific Nicolet iN 10Mx infrared microscope. ${ }^{1} \mathrm{H}$ NMR spectra was recorded on a $400 \mathrm{MHz}$ NMR spectrometer (Bruker DPX 400). Solid state ${ }^{13} \mathrm{C}$ NMR spectra were recorded on a Bruker Biospin GmbH. Ultraviolet-visible spectroscopy (UV-vis) spectra were collected using a Cary 100 spectrophotometer. Fluorescence measurements were collected using an Edinburgh Instruments FLS920P spectrofluorimeter. X-ray photoelectron spectroscopy (XPS) was performed on a Thermo Fisher Scientific ESCALAB 250 using monochromated Al K $\alpha$ X-rays (1486.6 $\mathrm{eV}$ ). Elemental analysis (EA) results were collected using a Thermo Scientific Flash Smart elemental analyzer OEA 2000. The apparent surface area of the final $\mathrm{CN}$ products was calculated by applying the Brunauer-Emmett-Teller (BET) model to the isotherm data points of the adsorption branch recorded on a Quantachrome NOVAtouch LX3 surface analyzer $\left(\mathrm{N}_{2}\right.$ at $\left.77 \mathrm{~K}\right)$.

\section{Hydrogen generation test under visible light}

Hydrogen evolution measurements were performed in a Schlenk flask thermally regulated with a cooling system and a white LED array as the irradiation source, with $3 \% \mathrm{Pt}$ as a co-catalyst and triethanolamine (TEOA) as a hole scavenger in a previously reported setup. ${ }^{9}$ Hydrogen evolution experiments were conducted under Ar atmosphere and a constant temperature of $25^{\circ} \mathrm{C}$. In a $\mathrm{H}_{2}$ evolution measurement, $15 \mathrm{mg}$ of the sample were placed inside a $50 \mathrm{~mL}$ Schlenk flask with a solvent mixture $(19 \mathrm{~mL})$ composed of water and TEOA in a $9: 1(\mathrm{v} / \mathrm{v})$ ratio and $19.6 \mu \mathrm{L}$ of a $\mathrm{H}_{2} \mathrm{PtCl}_{6}$ solution, (8\% in water). After stirring for $30 \mathrm{~min}$ in the dark under constant Ar flow, the reaction was started by switching on a $50 \mathrm{~W}$ (the illumination intensity on the photocatalyst surface is $10000 \mu \mathrm{mol} \mathrm{s} \mathrm{m}^{-1}$ ) white LED array (Bridge lux BXRA-50C5300; $\lambda>410 \mathrm{~nm}$ ). In order to confirm that the evolved gas is hydrogen, the headspace of the reactor was analyzed by Gas Chromatography (Agilent 7820 GC System). Quantum-yield measurements were performed in a sealed reactor connected to an argon line and an Agilent 7820 GC system. ${ }^{46}$ Argon gas was continuously flowing through the reactor in the dark to remove the existing hydrogen, nitrogen, oxygen and other gases and the purging process was monitored by automatically sampling every $11 \mathrm{~min}$. After the purge, a Thorlabs LED (405 nm) was turned on, the hydrogen produced was recorded and the integrated area was used for the calculation of the average quantum yield (AQY\%) which was performed as follows: $\mathrm{AQY}=N_{\mathrm{e}} / N_{\mathrm{p}} \times 100 \%=2 M / N_{\mathrm{p}}$ $\times 100 \%$, where $N_{\mathrm{e}}$ is the amount of reaction electrons, $N_{\mathrm{p}}$ is the incident photons, and $M$ is the amount of $\mathrm{H}_{2}$ molecules.

\section{Dye degradation under visible light}

The photocatalytic activity was further evaluated by the degradation of rhodamine $\mathrm{B}(\mathrm{RhB})$. In a typical photocatalytic degradation experiment $\mathrm{RhB}\left(20 \mathrm{~mL}, 20 \mathrm{mg} \mathrm{L}^{-1}\right)$ and carbon nitride $(20 \mathrm{mg})$ were mixed in a glass vial in the dark under continuous stirring until the adsorption-desorption equilibrium between the dye and the catalyst was obtained. After turning on the light, aliquots were withdrawn from the suspension at a given time interval. The concentration of the remaining dye in solution was monitored by optical absorption measurements (measured at $\lambda_{\max }=554$ ).

\section{Electrochemical measurements}

Electrochemical measurements were recorded using a threeelectrode system on an Autolab potentiostat (Metrohm, PGSTAT 101). A Pt-foil electrode and an $\mathrm{Ag} / \mathrm{AgCl}(3 \mathrm{M} \mathrm{KCl})$ electrode were used as the counter and reference electrodes, respectively. Photocurrent measurements and impedance spectroscopy were conducted with $\mathrm{KOH}(0.1 \mathrm{M})$ as electrolyte, saturated $\mathrm{Ag} / \mathrm{AgCl}$ as reference electrode and $\mathrm{Pt}$ as counter electrode. Photocurrents were measured at $1.23 \mathrm{~V}$ vs. RHE under the illumination of a solar simulator (Newport $300 \mathrm{~W}$ Xe arc lamp, equipped with air mass $\mathrm{AM} 1.5 \mathrm{G}$ and water filters) at the power density of $100 \mathrm{~mW} \mathrm{~cm}^{-2}$. For photoelectrochemical measurements, the working electrode was prepared by dispersing $10 \mathrm{mg}$ of $\mathrm{CN}$ materials into $4 \mathrm{~mL}$ of deionized water followed by sonication to get slurry. Then the slurry was drop casted onto cleaned FTO. After air-drying, the working electrode was further dried at $300{ }^{\circ} \mathrm{C}$ for $1 \mathrm{~h}$ to improve adhesion. Electrochemical impedance spectroscopy (EIS) was measured in dark and light (under illumination with a solar simulator with a power density of $100 \mathrm{~mW} \mathrm{~cm}^{-2}$ ). Nyquist plots of the prepared electrodes were measured in the frequency range from $100 \mathrm{kHz}$ to $10 \mathrm{MHz}$ at an applied voltage of $1.23 \mathrm{~V}$ vs. RHE. 


\section{Results and discussion}

Here, we took advantage of the tendency of melamine and cyanuric acid to preorganize in the interface between two solvents for tailoring new supramolecular assemblies. We envisioned that the bonding possibilities of creatine will allow its selective interaction with melamine and cyanuric acid populations in the interface between water and chloroform. Melamine (M) and creatine (C) were dissolved in water to form the MC complex. The interaction between both monomers was proven by ${ }^{1} \mathrm{H}-\mathrm{NMR}$, FTIR and XRD (discussion in Fig. S1 and S2. $\dagger$ We note that we focused on $1: 0.1 \mathrm{M}: \mathrm{C}$ ratio, $5 \mathrm{mmol}$ melamine and $0.5 \mathrm{mmol}$ creatine, due to the higher photocatalytic performance of the resulting material, as shown later in this paper). The formation of cyanuric acid-melamine derivatives was achieved by adding $25 \mathrm{~mL}$ of a cyanuric acid solution in chloroform to the MC solution in water. After certain time, the $\mathrm{CMC}_{x}$ supramolecular assembly is formed in the interface between both solvents (Scheme 1), and the solids are collected and dried in vacuum overnight.

The structural and chemical modifications induced by creatine in the cyanuric acid-melamine complex were studied by several means; FTIR (Fig. S3a†) proves the modification of the CM structure at high creatine concentrations, shown by the presence of typical vibrational modes of creatine, as the $\mathrm{O}-\mathrm{H}$, $\mathrm{C}-\mathrm{H}$ stretching vibrations coming from the carboxylic acid and methyl group, at $3201,3050 \mathrm{~cm}^{-1}$ respectively, $\mathrm{C}-\mathrm{O}$ stretching mode at $1088 \mathrm{~cm}^{-1}$ or $\mathrm{C}-\mathrm{H}$ bending vibration at $760 \mathrm{~cm}^{-1}$. The rearrangement in the crystal structure was confirmed by the XRD patterns (Fig. S3b $\dagger$ ), the strong lamellar stacking peak in the $\mathrm{CM}$ unit at $27.9^{\circ}$ disappears and three peaks at 17.6, 26.2 and 29.7 emerge, corresponding to the (110), (121), and (002) crystal planes. Elemental analysis (Table $\mathrm{S} 1 \dagger$ ) shows increasing $\mathrm{C} / \mathrm{N}$ ratios with higher creatine amounts $\left(0.6\right.$ in $\mathrm{CMC}_{0.25}$ vs. 0.51 in $\mathrm{CM}$ ), confirming its insertion within the structure. XPS measurements (Fig. S4 $\dagger$ ) further prove the chemical modifications in the $\mathrm{CM}$ lattice (for this analysis just $\mathrm{CM}$ and $\mathrm{CMC}_{0.1}$ were analysed due to the high photocatalytic performance of the corresponding carbon nitride, as shown later in this paper); C1s

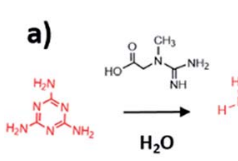

b)
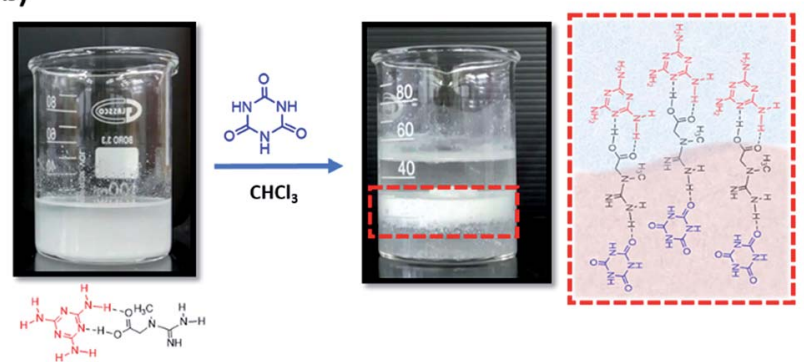

Scheme 1 Synthetic pathway of the CMC assemblies, (a) and illustration of the hydrogen bond generation in the interface (b). spectra of the CM complex shows four different chemical states at 284.8, 286.0, 287.8 and $289.7 \mathrm{eV}$, which correspond to $\mathrm{C}-\mathrm{C}$ and $\mathrm{C}-\mathrm{O}$ adventitious bonds, $\mathrm{C}-(\mathrm{N})_{3}$ from melamine units and $\mathrm{O}=\mathrm{C}-(\mathrm{N})_{2}$ from cyanuric acid, respectively. The formation of $\mathrm{CMC}_{x}$ complexes increases dramatically the atomic weight percentage of the $\mathrm{C}-(\mathrm{N})_{3}$ due to a synergistic contribution of melamine and creatine groups and as well the $\mathrm{C}-\mathrm{O}$, coming from the carboxylic group in creatine. N1s spectrum of CM displays three different binding energies at 398.8, 400.0 and $401.1 \mathrm{eV}$, that correspond to $\mathrm{C}=\mathrm{N}-\mathrm{C}$ of triazine rings, hydrogen bonded $\mathrm{NH}-\mathrm{O}$ groups, and remaining amino groups, respectively, the N1s spectra doesn't show pronounced differences, due to the similarity in the $\mathrm{N}$ species in the $\mathrm{CM}$ lattice and creatine. The morphology of the $\mathrm{CMC}_{x}$ assemblies, studied by SEM, shows that the insertion of creatine directs the growth of the supramolecular units towards one direction, resulting in micrometre-long aggregates, while the $\mathrm{CM}$ assembly in the absence of creatine results in a stone-like morphology (Fig. 1). Despite the previous report from our group proving that the utilization of an interfacial system for the synthesis of hydrogen-bonded frameworks strongly modifies their morphology, ${ }^{41}$ we wanted to make sure that in this work the preorganization in the interface between water and chloroform was providing a unique morphology. Hence, we synthesized the $\mathrm{CMC}_{0.1}$ complex just in water and analysed its morphology by SEM (Fig. S5 $\dagger$ ), confirming that its structural features strongly differ to the ones of $\mathrm{CMC}_{0.1}$ synthetized in an interfacial system. We observed that the size of the aggregates is considerably smaller in the absence of chloroform, probably due to the fact that the preorganization in between solvents allows a better rearrangement in plane, resulting in bigger stick-like aggregates (Fig. 1c).

The formation of graphitic carbon nitride materials was achieved by the pyrolysis of the supramolecular assemblies at $550{ }^{\circ} \mathrm{C}$ for $4 \mathrm{~h}$ under $\mathrm{N}_{2}$ atmosphere. We expect, that during the pyrolysis, the creatine motifs interlinking the cyanuric acidmelamine lattices condensate to form a guanidine-like motifs, $\left[\mathrm{HNC}\left(\mathrm{NH}_{2}\right)_{2}\right]$, which bind the heptazine units and modifies the properties of the material (Scheme $\mathrm{S} 1 \dagger$ ). ${ }^{47}$ The formation of $\mathrm{CN}-$
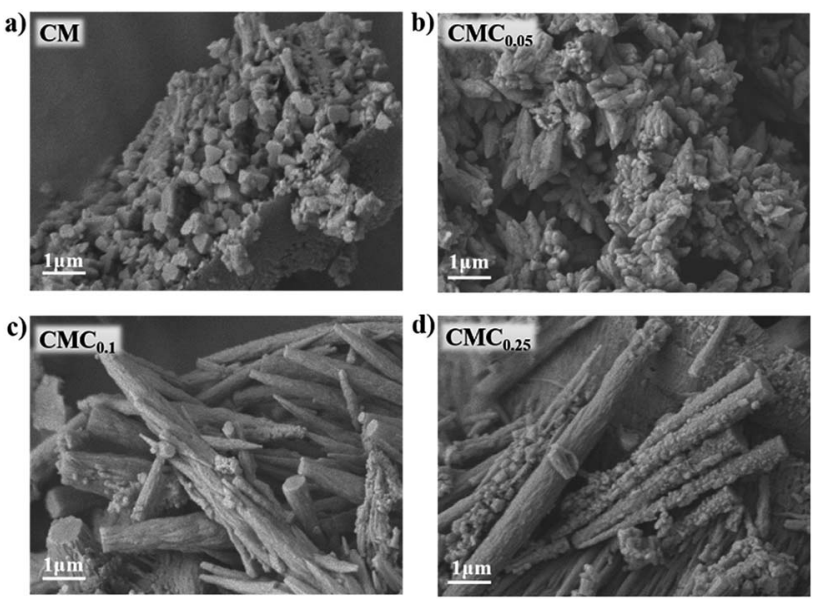

Fig. 1 SEM images of $\mathrm{CMC}_{x}$ supramolecular assemblies. 
like structures was confirmed by several means, FTIR shows the typical stretching vibrational modes of $\mathrm{C}-\mathrm{N}$ heterocycles between $1200-1600 \mathrm{~cm}^{-1}$ and the breathing modes of the triazine units at $810 \mathrm{~cm}^{-1}$ (Fig. S6†). Furthermore, XRD suggest the modification of the in-plane structure of the materials, as the peak at $13.1^{\circ}$, corresponding to the (100) plane, in CM-CN is shifted towards higher angles, probably due to the inclusion of molecular linkers within the heptazine units. Meanwhile the peak at $27.1^{\circ}$ which belong to the (002) crystal plane remains unmodified with higher concentrations of creatine in the starting assembly. XPS measurements (discussion in Fig. S7†) further prove the formation of carbon nitride-like materials. The morphology of the CN materials, studied by SEM (Fig. 2), shows that upon calcination the CM assembly turns into hollow tubes, as previously reported by our group, and the insertion of guanidine like units in the supramolecular assembly modifies it towards more hollow layers. TEM imaging (Fig. S8 $\dagger$ ) confirms also the formation of hollow tubes and thin nanosheets upon addition of higher creatine amounts. The increasing porosity in $\mathrm{CMC}_{x}$ is reflected also in the specific surface area as (from $54 \mathrm{~m}^{2}$ $\mathrm{g}^{-1}$ in $\mathrm{CM}-\mathrm{CN}$ to $75 \mathrm{~m}^{2} \mathrm{~g}^{-1}$ in $\mathrm{CMC}_{0.1}-\mathrm{CN}$, Fig. S9†).

The chemical environment of $\mathrm{C}$ atoms in $\mathrm{CM}$ and $\mathrm{CMC}_{0.1^{-}}$ $\mathrm{CN}$ were examined by ${ }^{13} \mathrm{C}$ CP-MAS NMR analysis (Fig. S10 $\dagger$ ). In both spectrum, two clear signals were observed around 164 and $157 \mathrm{ppm}$. The peak at $\sim 164 \mathrm{ppm}$ is ascribed to $\mathrm{C}$ atoms bonded to the external $-\mathrm{NH}_{2}$ groups $\left(\mathrm{N}_{2} \mathrm{C}-\mathrm{NH}_{2}\right.$ unit), whereas peaks at $\sim 157 \mathrm{ppm}$ are attributable to the $\mathrm{sp}^{2}$ species linked trigonally to three $\mathrm{N}$ atoms $\left(\mathrm{CN}_{3}\right)$ which stands for the presence of typical tri$s$-triazine or heptazine rings. The resolution of these peaks and the signal to noise ratio of ${ }^{13} \mathrm{C}$ NMR spectra become gradually lower indicating a feasible disturbance of the polymeric matrix due to the linking units created by the condensation of creatine.

The photophysical properties of the as-prepared materials were studied by UV-vis absorbance and photoluminescence measurements. This study revealed that the modifications imprinted within the carbon nitride networks change the electron distribution and alter the fundamental electronic/optical properties of resultant CN. UV-vis absorbance (Fig. 3a) shows

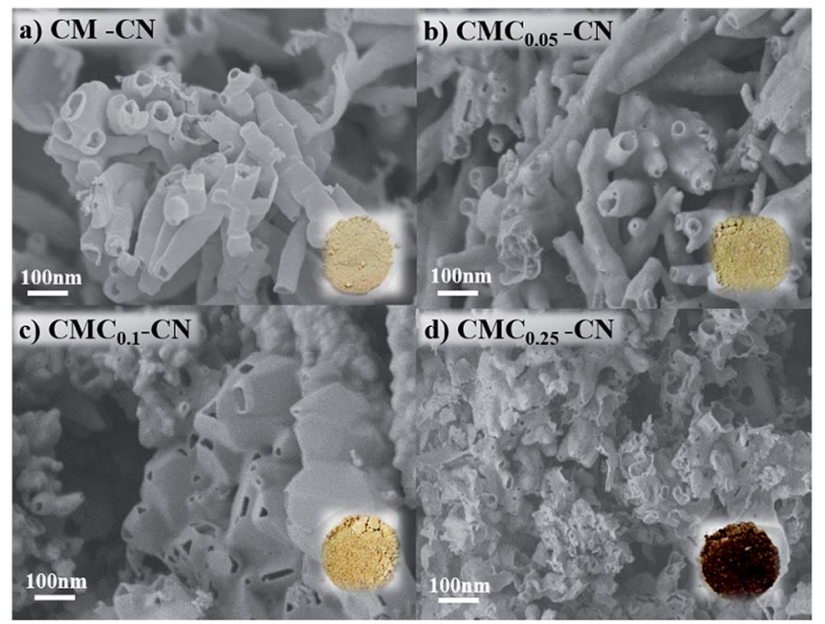

Fig. 2 SEM images of $\mathrm{CMC}_{x}-\mathrm{CN}$ materials. The insets show digital images of the $\mathrm{CMC}_{x}-\mathrm{CN}$ powders.
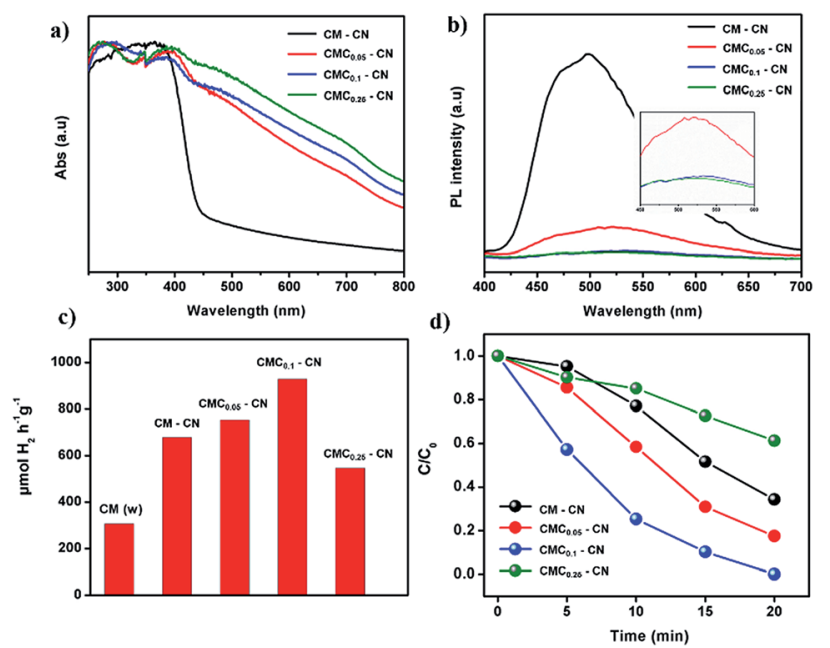

Fig. 3 UV-vis absorbance spectra (a), fluorescence spectra (b), hydrogen evolution performances (c), and RhB degradation (d) performances of $\mathrm{CM}-\mathrm{CN}$ and $\mathrm{CMC}_{x}-\mathrm{CN}$ materials.

the shift of the band corresponding to the $\pi-\pi^{*}$ excitation from 370 (in $\mathrm{CM}-\mathrm{CN})$ to $395 \mathrm{~nm}\left(\mathrm{CMC}_{x}-\mathrm{CN}\right)$ as well as the appearance of new peaks at 470 and $700 \mathrm{~nm}$ indicating that the resulting modified CN shows extended $\pi$-conjugation and enhanced light harvesting properties. Overall, with the increasing amount of creatine content, the $\mathrm{C} / \mathrm{N}$ ratio has steadily increased in the final materials which is confirmed by elemental analysis (Table S2 $†$ ). Photoluminescence measurements suggest a new, non-radiative path for photoexcited charges which result in the quenching in their fluorescence intensity (Fig. 3b). With all data in hand we can confirm that the monomer sequence alters the electronic structure of the final materials towards a more efficient charge separation properties, beneficial for photocatalysis.

The photocatalytic activity of the materials was tested by performing the hydrogen evolution reaction in a water/ triethanolamine solution with $\mathrm{Pt}$ as a co-catalyst (Fig. 3c), $\mathrm{CMC}_{0.1}$ showed the highest photocatalytic activity, resulting in $930 \mu \mathrm{mol} \mathrm{H} \mathrm{H}_{2} \mathrm{~h}^{-1} \mathrm{~g}^{-1}$ with a remarkable stability (92\% of the initial performance after three consecutive cycles, Fig. S11 $\dagger$ ) and an average quantum yield (AQY) at $405 \mathrm{~nm}$ of $2.6 \%$, this value is relatively high considering that no templating or ultrasonication techniques were employed. Additionally, the structure and morphology of the material remained intact after almost $15 \mathrm{~h}$ of photocatalytic hydrogen evolution. High resolution TEM images (HRTEM) of the material before and after the photocatalytic hydrogen evolution (Fig. S12 $\dagger$ ) show that the wrinkled nanosheet structure remains the same and just a slight decrease in the particle size is observed, and XPS measurements confirm as well that the chemical structure of the CN material remains undamaged (discussion in Fig. S13†). Further proof of the high photocatalytic activity was given by the degradation of the rhodamine $\mathrm{B}$ dye (RhB) under visible light (Fig. 3d), where $\mathrm{CMC}_{0.1}-\mathrm{CN}$ showed the fastest photodegradation performance $(20 \mathrm{~min})$.

In addition, the photocurrent responses of the $\mathrm{CM}-\mathrm{CN}$ and $\mathrm{CMC}_{0.1}-\mathrm{CN}$ films were measured in $0.1 \mathrm{M} \mathrm{KOH}$ at $1.23 \mathrm{~V} v s$. RHE under 1 sun illumination, where the $\mathrm{CMC}_{0.1}-\mathrm{CN}$ electrode 

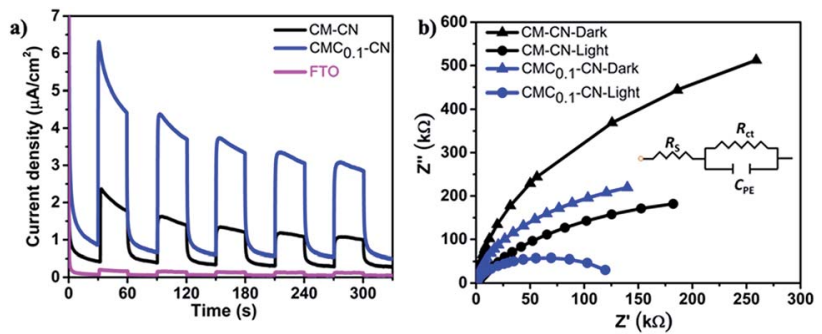

Fig. 4 Photocurrent response under visible light irradiation in $0.1 \mathrm{M}$ $\mathrm{KOH}$ at 1.23 (V) vs. RHE (a) and electrochemical impedance spectroscopy plots in the dark and under one sun illumination (b) (inset shows equivalent circuit for fitting the Nyquist plots).

exhibited the highest photocurrent response (Fig. 4a). The photocurrent results are further supported by electrochemical impedance spectra (EIS). The measurements are performed in dark and under one sun illumination, showing in both cases a lower charge transfer resistance for $\mathrm{CMC}_{0.1}-\mathrm{CN}$ (Fig. 4b).

\section{Conclusions}

In summary, we showed the formation of modified $\mathrm{CN}$ materials derived of a rationally designed supramolecular assembly. The utilization of a molecule that can bind selectively both melamine and cyanuric acid in the interface between water and chloroform allows a robust grafting and interlink of cyanuric acid-melamine hydrogen bonded frameworks. This new pathway strongly modifies the chemical and electronic properties of the final $\mathrm{CN}$ materials toward an efficient photocatalytic performance. We believe that this work represents a step forward in understanding the rational design of carbon-nitrogen based materials properties utilizing supramolecular structures and in the further establishment of structure-activity relationships. Additionally, it opens the gate towards the utilization of interfacial systems for the preorganization of supramolecular frameworks that modulate the performance of a given material in different energyrelated and environmental fields.

\section{Conflicts of interest}

The authors declare no conflict of interest.

\section{Acknowledgements}

The authors would like to thank Dr Michael Volokh Dr Alexander Upcher, and Mr Jonathan Tzadikov for assistance with electronic microscopy measurements and Prof. Menny Shalom for fruitful discussion. This research was funded by the Israel Science Foundation (ISF), grant No. 1161/17, and supported by the Minerva Center No. 117873.

\section{Notes and references}

1 W. J. Ong, L. L. Tan, Y. H. Ng, S. T. Yong and S. P. Chai, Chem. Rev., 2016, 116, 7159-7329.

2 J. Wen, J. Xie, X. Chen and X. Li, Appl. Surf. Sci., 2017, 391, 72-123.
3 J. Safaei, N. A. Mohamed, M. F. Mohamad Noh, M. F. Soh, N. A. Ludin, M. A. Ibrahim, W. N. Roslam Wan Isahak and M. A. Mat Teridi, J. Mater. Chem. A, 2018, 6, 22346-22380.

4 X. Wang, K. Maeda, A. Thomas, K. Takanabe, G. Xin, J. M. Carlsson, K. Domen and M. Antonietti, Nat. Mater., 2009, 8, 76-80.

5 M. Volokh, G. Peng, J. Barrio and M. Shalom, Angew. Chem., Int. Ed., 2019, 58, 6138-6151.

6 J. Lin, Z. Pan and X. Wang, ACS Sustainable Chem. Eng., 2014, 2, 353-358.

7 Y. Fang and X. Wang, Chem. Commun., 2018, 54, 5674-5687. 8 Q. Zheng, D. P. Durkin, J. E. Elenewski, Y. Sun, N. A. Banek, L. Hua, H. Chen, M. J. Wagner, W. Zhang and D. Shuai, Environ. Sci. Technol., 2016, 50, 12938-12948.

9 J. Barrio and M. Shalom, ACS Appl. Mater. Interfaces, 2018, 10, 39688-39694.

10 Z. Zhou, Y. Zhang, Y. Shen, S. Liu and Y. Zhang, Chem. Soc. Rev., 2018, 47, 2298-2321.

11 L. Chen and J. Song, Adv. Funct. Mater., 2017, 27, 1702695.

12 J. Xu, M. Shalom, F. Piersimoni, M. Antonietti, D. Neher and T. J. K. Brenner, Adv. Opt. Mater., 2015, 3, 913-917.

13 A. Savateev, I. Ghosh, B. König and M. Antonietti, Angew. Chem., Int. Ed., 2018, 57, 15936-15947.

$14 \mathrm{~J} . \mathrm{Xu}$ and M. Shalom, ChemPhotoChem, 2019, 3, 170-179.

15 L. Jiang, X. Yuan, Y. Pan, J. Liang, G. Zeng, Z. Wu and H. Wang, Appl. Catal., B, 2017, 217, 388-406.

16 X. Wu, F. Chen, X. Wang and H. Yu, Appl. Surf. Sci., 2018, 427, 645-653.

17 Z. Yang, Y. Zhang and Z. Schnepp, J. Mater. Chem. A, 2015, 3, 14081-14092.

18 J. Liu, J. Yan, H. Ji, Y. Xu, L. Huang, Y. Li, Y. Song, Q. Zhang, H. Xu and H. Li, Mater. Sci. Semicond. Process., 2016, 46, 59-68.

19 J. Fu, J. Yu, C. Jiang and B. Cheng, Adv. Energy Mater., 2018, 8, 1701503.

20 J. Barrio, C. Gibaja, J. Tzadikov, M. Shalom and F. Zamora, Adv. Sustainable Syst., 2019, 3, 1800138.

21 J. Wang, J. Chen, P. Wang, J. Hou, C. Wang and Y. Ao, Appl. Catal., B, 2018, 239, 578-585.

22 T. Wu, P. Wang, J. Qian, Y. Ao, C. Wang and J. Hou, Dalton Trans., 2017, 46, 13793-13801.

23 M. Shalom, S. Inal, D. Neher and M. Antonietti, Catal. Today, 2014, 225, 185-190.

24 Y. Zhang, T. Mori, J. Ye and M. Antonietti, J. Am. Chem. Soc., 2010, 132, 6294-6295.

25 J. Barrio and M. Shalom, ChemCatChem, 2018, 10, 55735586.

26 S. Wan, M. Ou, Q. Zhong, S. Zhang and W. Cai, Adv. Opt. Mater., 2017, 5, 1700536.

27 M. Shalom, M. Guttentag, C. Fettkenhauer, S. Inal, D. Neher, A. Llobet and M. Antonietti, Chem. Mater., 2014, 26, 58125818.

28 S. Guo, Z. Deng, M. Li, B. Jiang, C. Tian, Q. Pan and H. Fu, Angew. Chem., Int. Ed., 2016, 55, 1830-1834.

29 T. Jordan, N. Fechler, J. Xu, T. J. K. Brenner, M. Antonietti and M. Shalom, ChemCatChem, 2015, 7, 2826-2830. 
30 J. Barrio, L. Lin, P. Amo-Ochoa, J. Tzadikov, G. Peng, J. Sun, F. Zamora, X. Wang and M. Shalom, Small, 2018, 14, 1800633.

31 J. Wang, H. Xu, X. Qian, Y. Dong, J. Gao, G. Qian and J. Yao, Chem.-Asian J., 2015, 10, 1276-1280.

32 Y. S. Jun, E. Z. Lee, X. Wang, W. H. Hong, G. D. Stucky and A. Thomas, Adv. Funct. Mater., 2013, 23, 3661-3667.

33 Q. Li, J. Yang, D. Feng, Z. Wu, Q. Wu, S. S. Park, C.-S. Ha and D. Zhao, Nano Res., 2010, 3, 632-642.

34 J. Barrio, A. Grafmüller, J. Tzadikov and M. Shalom, Appl. Catal., B, 2018, 237, 681-688.

35 Q. Cui, J. Xu, X. Wang, L. Li, M. Antonietti and M. Shalom, Angew. Chem., Int. Ed., 2016, 55, 3672-3676.

36 X. Wu, D. Gao, H. Yu and J. Yu, Nanoscale, 2019, 11, 96089616.

37 P. Li, H. D. Arman, H. Wang, L. Weng, K. Alfooty, R. F. Angawi and B. Chen, Cryst. Growth Des., 2015, 15, 1871-1875.

38 V. R. Pedireddi and D. Belhekar, Tetrahedron, 2002, 58, 29372941.
39 J. Sun, J. Xu, A. Grafmueller, X. Huang, C. Liedel, G. AlgaraSiller, M. Willinger, C. Yang, Y. Fu, X. Wang and M. Shalom, Appl. Catal., B, 2017, 205, 1-10.

40 Q. Bo, Z. Shuai, S. Qiao, H. Zehuan, X. Jiang-Fei and Z. Xi, Angew. Chem., Int. Ed., 2017, 56, 7639-7643.

41 S. Dolai, J. Barrio, G. Peng, A. Grafmüller and M. Shalom, Nanoscale, 2019, 11, 5564-5570.

42 B. Roy, P. Bairi and A. K. Nandi, RSC Adv., 2014, 4, 17081734.

43 C. Li, B. J. Cafferty, S. C. Karunakaran, G. B. Schuster and N. V. Hud, Phys. Chem. Chem. Phys., 2016, 18, 20091-20096.

44 G. Li, J. Shi, G. Zhang, Y. Fang, M. Anpo and X. Wang, Res. Chem. Intermed., 2017, 43, 5137-5152.

45 M. Shalom, S. Inal, C. Fettkenhauer, D. Neher and M. Antonietti, J. Am. Chem. Soc., 2013, 135, 7118-7121.

46 P. Kalisman, Y. Nakibli and L. Amirav, Nano Lett., 2016, 16, 1776-1781.

47 B. Wang, T. Dong, A. Myrlie, L. Gu, H. Zhu, W. Xiong, P. Maness, R. Zhou and J. Yu, Green Chem., 2019, 21, 29282937. 